Apuntes Universitarios, 2021: 11(1), enero-marzo

ISSN: 2304-0335 DOI: https://doi.org/10.17162/au.v11i1.547

\title{
Efectos del cambio climático en fincas cafetaleras: una revisión bibliográfica con énfasis en Perú
}

\author{
Effects of climate change on coffee farms: A literature review with an \\ emphasis on Peru
}

\begin{abstract}
Eli Morales Rojas ${ }^{1 \mathrm{a}}$; Segundo Chavez Quintana ${ }^{2}$ Jaris Veneros Guevara ${ }^{3}$; Edwin Díaz Ortiz Tito Sánchez Santillan ${ }^{5}$; Magali García Rosero ${ }^{6}$

Instituto de Investigación para el Desarrollo sustentable de Ceja de Selva INDES-CES,

\section{Amazonas ${ }^{1}$}

Universidad Nacional Toribio Rodríguez de Mendoza de Amazonas, Perú 2346

Universidad Estatal de Montana, Departamento de Ecología, Estados Unidos ${ }^{4}$

Instituto de Investigaciones de la Amazonía Peruana, Loreto, Perú. ${ }^{5}$
\end{abstract}

(iD) Orcid ID: https://orcid.org/0000-0002-8623-31921

Orcid ID: https://orcid.org/0000-0002-0946-3445

D Orcid ID: https://orcid.org/0000-0001-6981-40783

iD Orcid ID: https://orcid.org/0000-0001-7213-9552 4

iD Orcid ID: https://orcid.org/0000-0002-3352-341X

(iD) Orcid ID: https://orcid.org/0000-0001-7508-7516 6

Recibido: 27 de junio de 2020

Aceptado: 05 de octubre de 2020

\section{Resumen}

Los efectos del cambio climático están generando inestabilidad en la agricultura. En este sentido el propósito de este artículo de revisión bibliográfica es dar a conocer los efectos que está causando el cambio climático en fincas cafetaleras de pequeños agricultores de Perú, así como las alternativas de solución. Se concluye que el cambio climático está afectando negativamente a los cultivos de café, a través de la introducción de enfermedades tales como la roya en el año 2013 y 2014 disminuyo la producción de café en 109.500 toneladas (t), el afecto incidió a la variedad de café typica. En consecuencia, las familias han tenido que adaptarse a convivir con la roya, con una tendencia de implementar nuevas parcelas de café, sembradas por encima de los (1500 nsnm). Así mismo el precio del café es inestable, para el año 2013-2014 se mantuvo S/ 6.33 a S/ 4.89 por kg. 
Frente a las dificultades que hubo, el estado peruano ha incentivado a la siembra de café (catimor) desde el 2014 hasta el año 2018, se incrementó en 79.755 hectáreas de cultivo.

Palabras claves: Café, producción, tendencia, cambio climático, Perú.

\begin{abstract}
The effects of climate change are creating instability in agriculture. In this sense, the purpose of this literature review article is to publicize the effects that climate change is causing on smallholder coffee farms in Peru and to describe the methods used to determine global warming, as well as alternative solutions. It is concluded that climate change is negatively affecting coffee crops, by the introduction of diseases such as rust in 2013 and 2014 decreased coffee production by 109. 500 tons $(\mathrm{t})$, the effect affected the variety of coffee typica. Consequently, families have had to adapt and live with the rust; Thus, new coffee plots have been implemented, planted at higher altitudes (1500 nsnm). Likewise, the price of coffee is unstable, for the year 2013-2014 it remained S / 6.33 to $\mathrm{S} / 4.89$ per $\mathrm{kg}$. Faced with the difficulties that existed, the Peruvian state has encouraged the planting of coffee (catimor) from 2014 to 2018, increased by 79,755 hectares of cultivation.
\end{abstract}

Keywords: Coffee, production, trend, climate change, Peru.

\title{
Introducción
}

El cambio climático está acarreando grandes consecuencias en las actividades económicas de la población y los ecosistemas (IPCC, 2014). A ello se suma la trayectoria de emisiones de gases de efecto invernadero. Siendo los países de América Latina y el Caribe los más afectados (CEPAL, 2014). La incertidumbre por conocer el efecto que tendrá el cambio climático sobre la producción agrícola a nivel mundial y nacional es grande. El cambio climático en los cultivos de café, afecta las zonas empinadas y montañosas, generando cambios en la economía de los pequeños caficultores (Ramírez et al., 2015). Asimismo, estudios de análisis cualitativos muestran que el cambio climático ha ocasionado un incremento de la temperatura, generando variaciones en las precipitaciones (Brigido, 2015). Otros estudios realizados mediante modelos climáticos mencionan que las regiones experimentarán incrementos de temperatura y aumento de eventos climáticos extremos (CEPAL, 2014), donde el sector agropecuario en los países de América Latina y el Caribe son sensibles a estos efectos. El incremento de la temperatura, afectará el cultivo de café y sufrirá una redistribución geográfica, afectando a las zonas de latitudes y altitudes más bajas (Schroth et al., 2009; Ovalle-Rivero et al., 2015).

El cambio climático afectará la distribución, producción y rentabilidad del cultivo de café en América Latina, impactando la economía de los productores (Bunn et al., 2015). Existe diferentes alternativas de solución que demuestran que el uso de la biodiversidad y los servicios 
ecosistémicos es una solución al cambio climático, incrementando la producción de calidad (Vignola et al., 2015). En este sentido, Vallejo, Chacón y Cifuentes (2016), hablan de la utilidad de incentivar la mitigación y adaptación, con la finalidad de optimizar procesos desde su planteamiento hasta la implementación, logrando una mayor efectividad en los beneficios. En este contexto, el objetivo fue realizar una revisión bibliográfica de los efectos del cambio climático en café y las alternativas de solución con énfasis en Perú.

\section{Materiales y Métodos}

La revisión bibliográfica se realizó durante los meses de abril y mayo del 2020, utilizando la combinación de operadores booleanos y almacenamiento de artículos de revistas de alto impacto (Scielo, Directory of Open Access Journals (DOAJ), ScienceDirect). Y la revisión de publicaciones por el Ministerio del Ambiente (MINAM - PERÚ), Ministerio de Agricultura y Riego del Perú (MINAGRI) y el Sistema Integrado de Estadística Agraria (SIEA). La referenciación se realizó con el gestor de referencias bibliográficas (Zotero), de acceso libre y gratuito. Se tuvo en cuenta la fecha de publicación de los artículos, preferentemente la revisión de artículos publicados en los últimos años.

\section{Revisión bibliográfica}

Los cambios en fincas cafetaleras años atrás era un asunto manejable, dado que la presencia de enfermedades era baja, sin embargo, las intensas lluvias hacen que haya más humedad, proliferando mayor cantidad de hongos, perdida de hojas en las plantaciones (Sánchez, 2018). El cambio climático ha traído consecuencias negativas sobre la producción de café, principalmente en plagas y enfermedades que antes no estaban evidenciadas y además han cambiado su comportamiento, volviéndose resistentes a diferentes controladores, generando mayor inversión en productos químicos (Velásquez, 2019).

El cambio climático ha ocasionado el aumento de los costos de producción y la renovación de los cafetales. Esto afecta directamente a las familias que poseen en promedio una hectárea y media de cultivo (Pérez, 2019), en muchas regiones la economía campesina depende del café, sin embargo los últimos tres decenios las consecuencias del cambio climático acarreado el incremento de plagas y enfermedades, el bajo rendimiento; generando la rotación de los cultivos a los 2,300 msnm, lo que antes se cosechaba a los 1,500 m, de altitud (Mora, 2019). La producción de café ha 
sido afectada por la variabilidad climática, y la tendencia de los impactos del cambio climático podrían ser más severos con el transcurrir del tiempo, provocando una disminución de cultivos en altitudes más bajas y latitudes más altas, con la probabilidad de disminuir hasta en un 30\% de áreas de cultivo del café para el año 2050 (Jha et al., 2011; Baca et al., 2014).

\section{Tendencias observadas del cambio climático en Perú}

A consecuencia del cambio climático se estima una caída de entre 8 y $13 \%$ de los ingresos esperados económicos por hectárea, incrementándose la probabilidad de elección del café. Como es sabido la producción del café a mayor temperatura, menor nivel de producción (Galindo, 2015; Figueroa, 2018).

El cambio climático afecta negativamente a la canasta familiar, en aquellos agricultores que cuentan con pequeñas áreas de cultivos a diferentes pisos ecológicos, por ello se ven obligados a talar más terrenos, tal ejemplo la zona nororiental que consta de tres regiones, San Martín, Amazonas y Cajamarca. Siendo la región de San Martin la más deforestada por la expansión de cultivos de café y la palma aceitera (Zárate, 2016).

En Amazonas, la pérdida de bosque se concentra en las provincias de Rodríguez de Mendoza (15. 197 ha), y Bagua (13.162 ha) (MINAM, 2015). Y ocupa el $83 \%$ de la superficie por monte y bosques, seguido por pastos naturales ( $5 \%$ ), cultivos permanentes (4\%), el café es el principal cultivo permanente (28.377 ha) (INEI, 2012). Cajamarca, sus tierras está compuesto por cultivos permanentes $(16 \%)$ y purma $(12 \%)$ y el café es el principal cultivo permanente (62.710 ha) (INEI, 2012). Existe una relación directa entre la expansión de la superficie de café cultivada y la progresión de la deforestación. Debido a al incremento de las temperaturas por encima a los $30{ }^{\circ} \mathrm{C}$, las heladas durante semanas pueden llegar a matar la planta, incidencia de plagas, enfermedades y reducir el rendimiento (Wintgens, 2009). Los vientos fuertes (50 km/h) afectan el café, dañando ramas, hojas, flores, frutos y la erosión de suelos. Es así que el impacto se refleja en la pérdida de la calidad de café en taza (Robiglio, 2017). 
Figura 1.

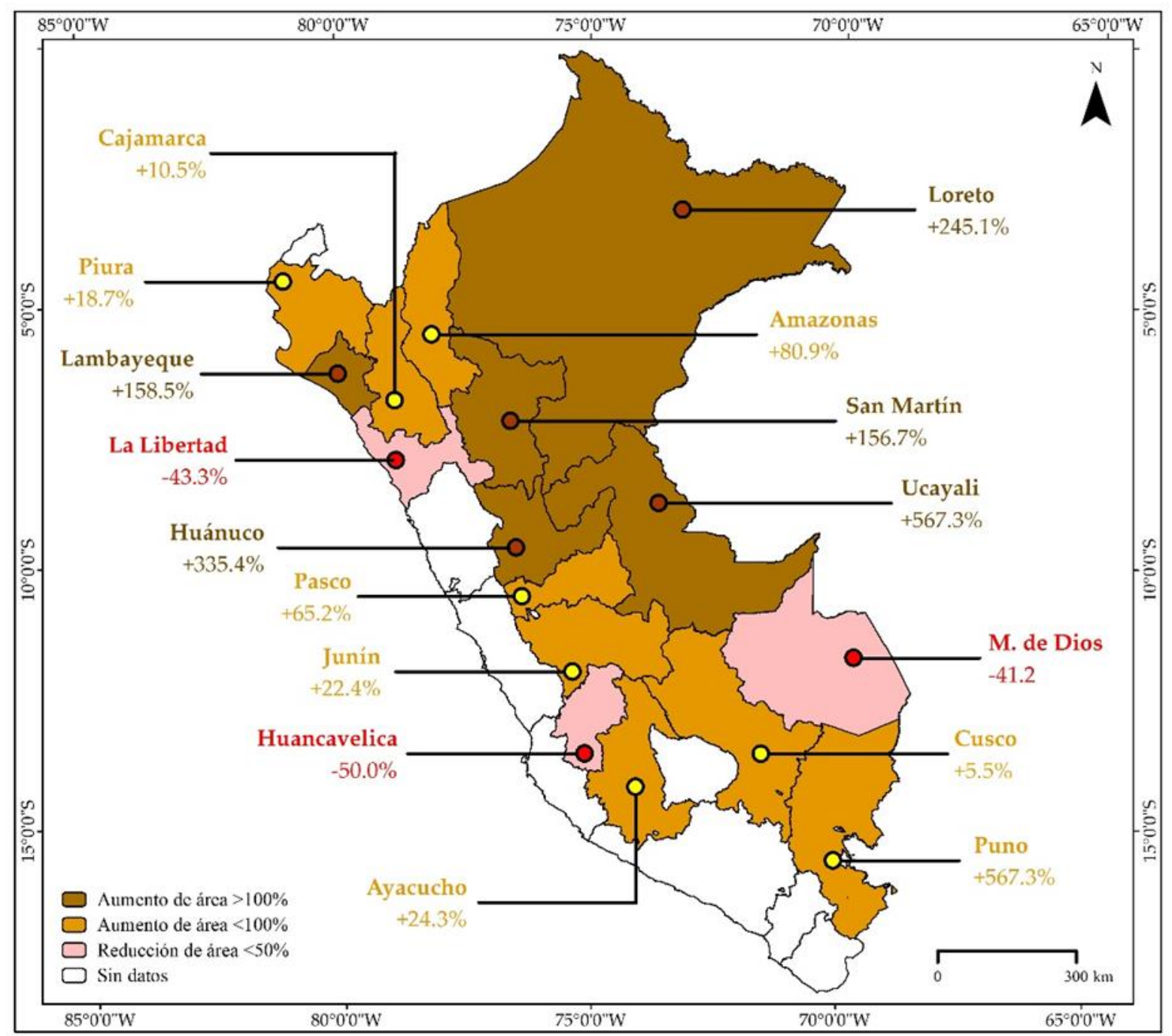

Figura 1. Mapa de distribución geográfica de superficie cultivada de café por regiones. (Aumento de áreas y reducción de áreas).

En la (Figura 1) se observa los cambios por traslación de parcelas de cultivos de café para todas las regiones productoras, evidenciándose una gran disminución en la región de Madre de Dios, Huancavelica y la Libertad. Con respecto a las demás regiones han aumentado el área de cultivo.

\section{Cambio climático en la fenología del café}

La fenología es el estudio temporal de las fases que atraviesa un organismo durante su ciclo de vida en las plantas (Bustamante et al., 2015); estudios han resaltado que el cambio climático en 
los últimos 30 años ha generado un impacto en la distribución, abundancia, fenología y fisiología de muchas especies (Jarvis et al. 2008). Las altas temperaturas, reducen la fotosíntesis neta en plantas, debido al incremento en las pérdidas de carbono (Wayne, 2002). En el cultivo de café, la distribución de los períodos húmedos y secos y la temperatura son las que determinan las etapas fenológicas, teniendo en cuenta que el periodo crítico es durante el desarrollo del fruto (Coa et al., 2015).

\section{Enfermedades asociadas al café en Perú}

El cambio climático está ocasionando la disminución en el rendimiento del café y en otras oportunidades aumenta la incidencia de las enfermedades tales como la roya, provocando incertidumbre en el calendario agrícola (Altea, 2020; Lechthaler, 2017). El 95\% de la producción de café proviene de la variedad arábica y es susceptible a enfermedades de la roya, ocasionando pérdidas hasta del 40\% en la producción (Quispe, 2017). La roya ha destruido 94 mil hectáreas de cafeto, teniendo efectos negativos en la producción entre los años 2013 y 2014.

El aumento de la producción café en el 2018 se debe a la inclusión de unas 15 mil hectáreas de cafetales plantadas en el 2015, sumado a ello 10 mil hectáreas nuevas de cafetos. En enerojunio del 2019 se ha logrado producir un volumen 267.8 mil toneladas, $1.2 \%$ menor al registrado en el mismo período del 2018 (271 mil toneladas). Es así que, la producción nacional tiene un comportamiento inestable, bajo los efectos climáticos, asociado al ataque de enfermedades. La roya es una enfermedades del café y su agente causal es el hongo biotrófico, parasito que necesita de células vivas para sobrevivir y completar su ciclo de vida (Quispe, 2017). Otra de las enfermedades que ataca al café es el ojo de gallo causado por Mycena citricolor, (Granados, 2020). La broca es una amenaza en el fruto del café, y está asociada con la altitud, se incrementa cuando está por debajo de los 1500 msnm (Rebolledo et al. 2016).

\section{Comportamiento de la producción regional en Perú}

El comportamiento de la producción regional es muy variado en estos últimos años: San Martín ha aumentado su producción de (34758 a 63893 t) y Junín (33109 a 89837 t), ambas han incrementado su producción. Así también como las demás regiones de Cajamarca (50336 a 63893 t), Amazonas (24227 a 43946 t), Cuzco (26333 a 30754 t), San Martin (34758 a 91423 t) (Puno 6147 a 7784 t), Huánuco (2012 a 10782 t), Junín (33109 a 89837 t), Ucayali (1199 a 8325 t), Pasco 
(3182 a 13610 t), Lambayeque (749 a 1748 t), Piura (2048 a 3660 t), y Loreto (72 a 178 t), han aumentado su producción al 2018 (Tabla 1; Figura 2). 
Tabla 1

Evolución ascendente de la producción de café pergamino en toneladas por regiones desde el año 2005-2018

\begin{tabular}{|c|c|c|c|c|c|c|c|c|c|c|c|c|}
\hline Años & Cajamarca & Amazonas & Cuzco & San Martin & Puno & Ucayali & Pasco & Lambayeque & Piura & Loreto & Junín & Huanuco \\
\hline 2005 & 50336 & 24227 & 26333 & 34758 & 6147 & 1199 & 3182 & 749 & 2048 & 72 & 33109 & 2012 \\
\hline 2006 & 55975 & 35059 & 44848 & 39334 & 7431 & 1352 & 6387 & 485 & 2621 & 66 & 73043 & 2009 \\
\hline 2007 & 54086 & 33353 & 21875 & 39313 & 5749 & 1450 & 5181 & 441 & 2186 & 82 & 55582 & 1949 \\
\hline 2008 & 55689 & 30205 & 35623 & 44473 & 5784 & 1702 & 7900 & 526 & 2929 & 83 & 82053 & 2066 \\
\hline 2009 & 57272 & 31812 & 20502 & 48644 & 6393 & 1797 & 5818 & 406 & 3079 & 66 & 60792 & 2142 \\
\hline 2010 & 59020 & 35066 & 39069 & 52915 & 6084 & 2572 & 6706 & 595 & 2390 & 44 & 67790 & 2168 \\
\hline 2011 & 65051 & 35528 & 53548 & 63757 & 6452 & 2963 & 7847 & 509 & 2248 & 84 & 86519 & 2343 \\
\hline 2012 & 64901 & 38317 & 35730 & 68712 & 7364 & 3431 & 9596 & 431 & 1915 & 127 & 76714 & 2504 \\
\hline 2013 & 54472 & 32857 & 38545 & 47872 & 6832 & 2587 & 7197 & 675 & 2280 & 140 & 54837 & 2854 \\
\hline 2014 & 48682 & 33123 & 28426 & 56823 & 4750 & 5650 & 5434 & 533 & 2315 & 139 & 30202 & 3503 \\
\hline 2015 & 46083 & 35101 & 18413 & 82164 & 6504 & 5442 & 6898 & 863 & 2677 & 150 & 39275 & 5109 \\
\hline 2016 & 48182 & 34966 & 27163 & 82319 & 6940 & 4529 & 10094 & 1703 & 3044 & 162 & 46692 & 7850 \\
\hline 2017 & 62863 & 38893 & 26615 & 91197 & 7754 & 4004 & 11669 & 1553 & 4050 & 171 & 75100 & 9427 \\
\hline 2018 & 63893 & 43946 & 30754 & 91423 & 7784 & 8325 & 13610 & 1748 & 3660 & 178 & 89837 & 10782 \\
\hline $\begin{array}{c}\text { Prome } \\
\text { dios }\end{array}$ & 56179 & 34461 & 31960 & 60265 & 6569 & 3357 & 7680 & 801 & 2674 & 112 & 62253 & 4051 \\
\hline
\end{tabular}

Fuente: Ministerio de Agricultura y Riego (MINAGRI)- Dirección de Estadística Agraria de la Dirección General de Seguimiento y

Evaluación de Políticas (DGESEP). 
Figura 2

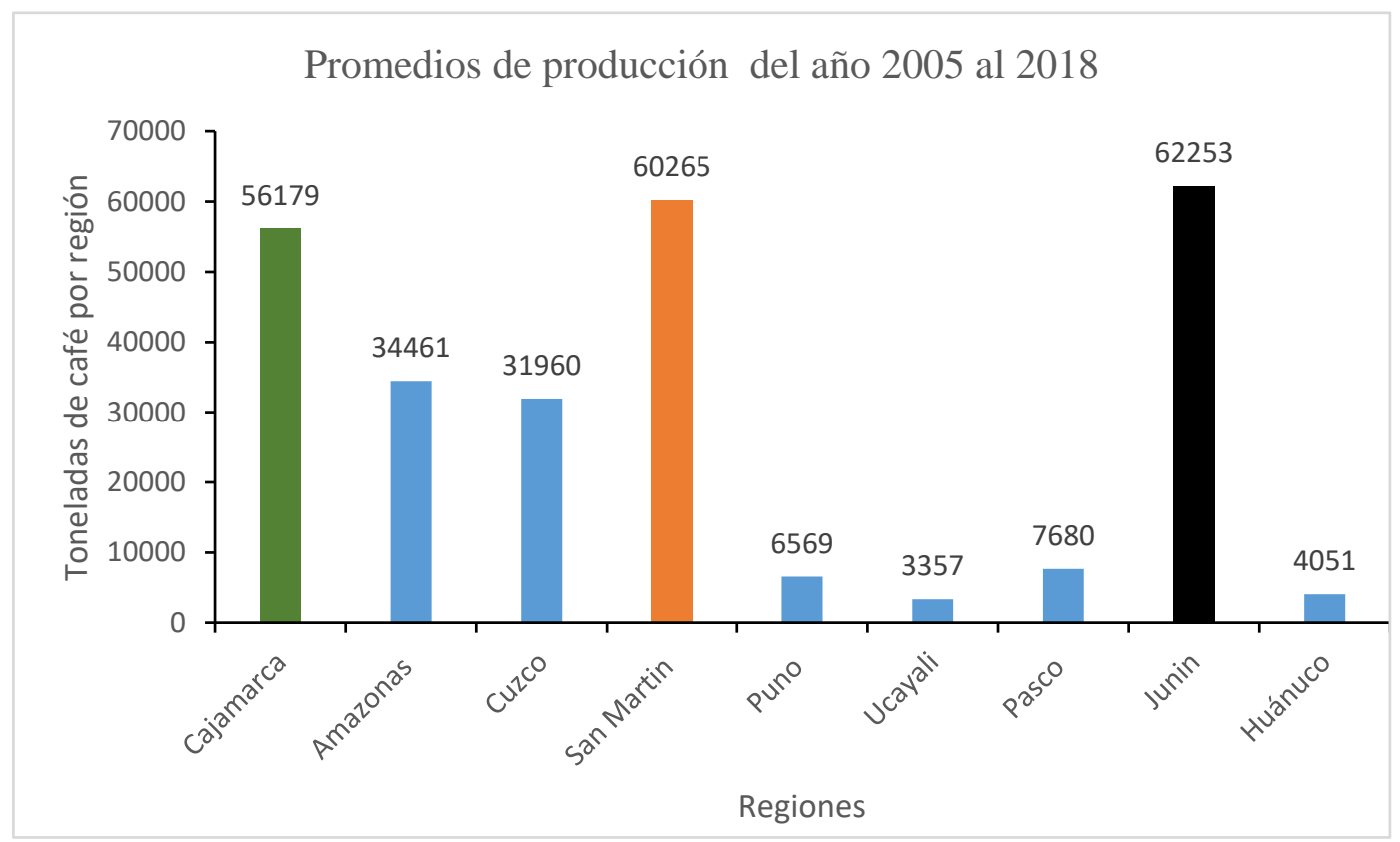

Figura 2. Promedios de producción de las principales regiones productoras (MINAGRI-DGESEP).

Mientras que otras regiones ha disminuido la producción en toneladas entre ellas esta Ayacucho (4110 a 3430 t), Apurímac (2 a 0 t), La Libertad (285 a 225 t), la región Lima evidencia que en el 2014 ha producido unas 60 toneladas. Sin embargo, para el 2018 ha sufrido una disminución a 51 toneladas (Tabla 2; Figura 3).

Tabla 2

Evolución descendente de la producción de café pergamino en toneladas por regiones desde el año 2005-2018

\begin{tabular}{cccccc} 
Años & Ayacucho & Huancavelica & La Libertad & Lima & M. de Dios \\
\hline 2005 & 4110 & 9 & 285 & 0 & 35 \\
2006 & 4221 & 8 & 303 & 0 & 35 \\
2007 & 4435 & 6 & 270 & 0 & 33 \\
2008 & 4423 & 10 & 271 & 0 & 44 \\
2009 & 4405 & 13 & 301 & 0 & 36 \\
2010 & 4441 & 8 & 303 & 0 & 29 \\
2011 & 4373 & 7 & 297 & 0 & 20 \\
2012 & 4402 & 7 & 301 & 0 & 18 \\
2013 & 4434 & 7 & 254 & 0 & 16 \\
2014 & 2273 & 7 & 177 & 69 & 12 \\
2015 & 3051 & 7 & 188 & 181 & 13 \\
\hline
\end{tabular}




\begin{tabular}{rccccc}
\hline 2016 & 3875 & 12 & 215 & 153 & 14 \\
2017 & 3781 & 12 & 227 & 155 & 14 \\
2018 & 3430 & 12 & 225 & 51 & 13 \\
Promedio & 3975 & 9 & 258 & 44 & 24 \\
\hline
\end{tabular}

Fuente: Ministerio de Agricultura y Riego (MINAGRI)- Dirección de Estadística Agraria de la Dirección General de Seguimiento y Evaluación de Políticas (DGESEP).

Figura 3

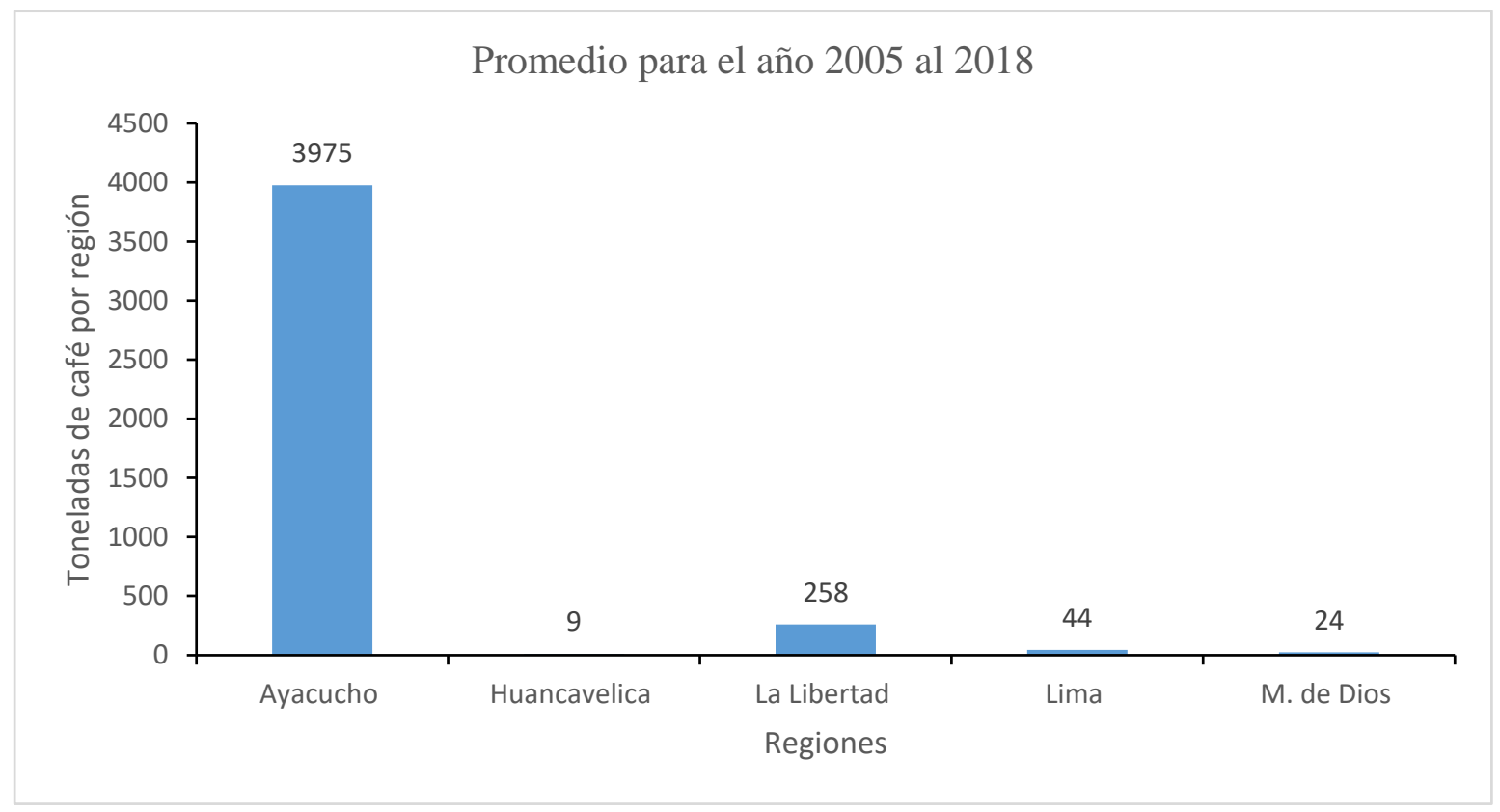

Figura 3. Promedios de producción de las principales regiones que disminuyo la producción de café pergamino (MINAGRI-DGESEP).

Las regiones que disminuyeron notablemente su producción son las que ya no han podido recuperarse después del impacto ocasionado por la roya en el año 2013. Del mismo modo se puede evidenciar que las regiones que se han mantenido en su producción oh han aumentado es debido a la expansión de nuevas parcelas (Zárate, 2016). Del mismo modo, la inestabilidad en los precios del café genera incertidumbre en los agricultores cafetaleros, tal es así que para el año 2011 llego a costar el kilo de café pergamino a 9.2 soles (Figura 4), mientras que para el 2013, disminuyo a 4.89 soles el kilo (Díaz et al, 2018). 
Figura 4

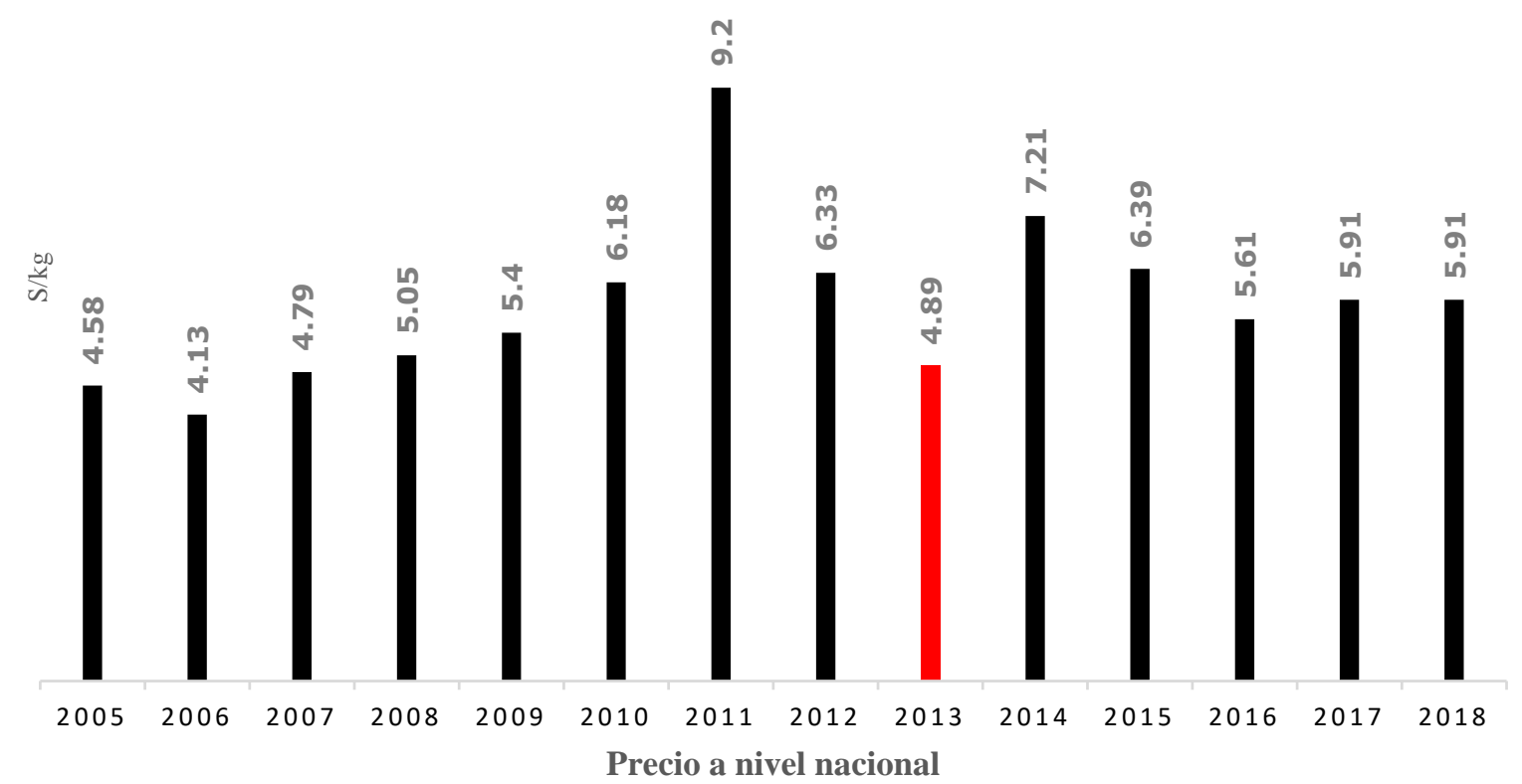

Figura 4. Variación del precio a nivel nacional de café en pergamino 2005-2018 (MINAGRI-DGESEPDEA).

\section{Métodos de evaluación del efecto del cambio climático}

Villers et al. (2009) propuso un método para evaluar los impactos del cambio climático, mediante diagramas ombrotérmicos, relacionando las variables de temperatura y precipitaciones mensuales en las etapas de floración y fructificación del café. Así mismo existen estudios de sistemas complejos, que integran al hombre-naturaleza, mediante la aplicación de la Lógica Difusa, con la finalidad de determinar la vulnerabilidad por el cambio climático (Gómez, 2015). Los estudios de evaluación de impactos provenientes del cambio climático en la agricultura, se realiza en base al modelo HadGEM2-ES para el forzamiento radiativo 8.5 en un futuro cercano, 20152039 (Montiel, 2017). La utilización del software ArcGis, para crear mapas de distribución geográfica y calcular áreas de distribuciones actuales, y los cambios climáticos en escenarios futuros para los años 2040, 2060 y 2080 (Quipuscoa, 2019).

\section{Alternativas de solución frente al cambio climático}

Para contrarrestar los impactos del cambio climático está en generar prácticas de manera conjunta del medio en donde viven, y compartir conocimientos que puedan ser aplicados por los 
productores (Chain, 2019). Las prácticas tecnológicas tales como la introducción de sistemas de riego, el uso de variedades mejoradas y la incorporación de elementos socioculturales y económicos son importantes para lograr un desarrollo sostenible (Gutiérrez, 2008)

Las prácticas agroforestales y agroecológicas son las más adecuadas para la adaptación frente al cambio climático y de fácil adopción por los productores agrícolas, dado que reducen el impacto del cambio climático y los eventos climáticos extremos (Altieri et al., 2015). La sombra de bosques naturales las defensas rivereñas, regula el flujo de cuerpos de agua bajo las condiciones cambiantes de lluvias (Capon et al., 2013), por otro lado conservación de bosques en áreas montañosas ayuda a prevenir la erosión y los deslizamientos (Locatelli et al., 2011). En los últimos cinco años, se reportó que los agricultores de la región San Martin, adaptaron la sombra con especies forestales como respuesta a las presiones de las plagas y enfermedades (Jezer, 2019). Prácticas para mitigar los impactos causado por los vientos es la adopción de cortinas rompe vientos. Mediante la implementación de cercas vivas (Tobar, 2010). El gobierno peruano ha venido promoviendo a las organizaciones cafetaleras para para la producción de café certificados y cafés especiales. Aunando estrategias mediante el acceso a créditos para la renovación de fincas (Libert, 2018), incentivando a la conservación de bosques, evitando la tala en la agricultura.

\section{Conclusiones}

El efecto del cambio climático se ve reflejado en agricultores que cultivan por debajo de los $1500 \mathrm{msnm}$, por el incremento de lluvias con mayor intensidad e inundaciones, enfermedades y la inestabilidad de precio del café. Dando lugar a la apertura de nuevas fincas de café por encima de los 1500 msnm. Se revisó los principales métodos de evaluación de los efectos del cambio climático en café entre ellos tenemos la aplicación de la lógica difusa, diagramas ombrotérmicos que permiten relacionar las variables de temperatura y precipitación. Asimismo, se puede predecir la variabilidad de estudios de proyecciones predictivas de los efectos que puede causar el cambio climático a 50 años, aplicando programas de ArcGIS. Finalmente se promueven plantaciones de sombra en el café, dado proporcionan un refugio a los seres vivientes, a la vez que protegen las plantas de café durante los cambios meteorológicos extremos, estas actúan como barreras para el viento durante las lluvias y tormentas ayudando a reducir la erosión de los suelos. 


\section{Referencias}

Altea, L. (2020). Perceptions of climate change and its impacts: a comparison between farmers and institutions in the Amazonas Region of Peru. Climate and Development, 12(2), 134-146. doi: https://doi.org/10.1080/17565529.2019.1605285

Altieri, M. A., Nicholls, C. I., Henao, A., \& Lana, M. A. (2015). Agroecology and the design of climate change-resilient farming systems. Agronomy for sustainable development, 35(3), 869890. Obtenido de: https://link.springer.com/article/10.1007/s13593-015-0285-2

Bustamante González, C., Pérez Díaz, A., Rivera Espinosa, R., Martín Alonso, G. M., \& Viñals Nuñez, R. (2015). Influencia de las precipitaciones en el rendimiento de Coffea canephora Pierre ex Froehner cultivado en suelos Pardos de la región oriental de Cuba. Cultivos Tropicales, 36(4), 21-27. Obtenido de: http://scielo.sld.cu/scielo.php?pid=S025859362015000400003\&script=sci_arttext\&tlng=en

Brigido, J. G., Nikolskii, I., Terrazas, L., \& Herrera, S. S. (2015). Estimación del impacto del cambio climático sobre fertilidad del suelo y productividad de café en Veracruz, México. $\begin{array}{lllll}\text { Tecnología } y \quad \text { ciencias del } & \text { agua, }\end{array}$ http://www.scielo.org.mx/scielo.php?script=sci_arttext\&pid=S2007-24222015000400007

Bunn, C., P. Läderach, O. Ovalle-Rivera, and D. Kirschke. (2015). A bitter cup: climate change profile of global production of Arabica and Robusta coffee. Climatic Change 129:89-101. doi:10.1007/s10584-014-1306-x

Baca, M., P. Läderach, J. Haggar, G. Schroth, and O. Ovalle. (2014). An integrated framework for assessing vulnerability to climate change and developing adaptation strategies for coffee growing families in Mesoamerica. PLoS One 9:e88463. doi:10.1371/journal.pone.0088463

Capon, S. J., Chambers, L. E., Mac Nally, R., Naiman, R. J., Davies, P., Marshall, N. \& Catford, J. (2013). Riparian ecosystems in the 21st century: hotspots for climate change adaptation? Ecosystems, 16(3), 359-381. doi: https://doi.org/10.1007/s10021-013-9656-1

CEPAL (2014), La economía del cambio climático en América Latina y el Caribe: Paradojas y desafíos del desarrollo sostenible, CEPAL, Santiago de Chile. obtenido de: https://repositorio.cepal.org/handle/11362/37471

Chain-Guadarrama, A., Martínez-Rodríguez, M. R., Cárdenas, J. M., Vílchez-Mendoza, S., \& Harvey, C. A. (2019). Uso de prácticas de Adaptación basada en Ecosistemas por pequeños cafetaleros en Centroamérica. Agronomía Mesoamericana, 30(1), 1-18. doi: 
http://dx.doi.org/10.15517/am.v30i1.32615

Coa, M.; R. Silva-Acuña; J. Méndez \& S. Mundarain. 2015. Fenología de la floración del cafeto var. Catuaí Rojo en el Municipio Caripe del estado Monagas, Venezuela. IDESIA (Chile) 33(1):59-67. http://dx.doi.org/10.4067/S0718-34292015000100007

Díaz-Rodríguez, A., Silva-Jaimes, M. I., \& Dávila-Romero, J. C. (2018). Relación entre las buenas prácticas de higiene y la ocurrencia de ocratoxina A en café (Coffea arabica L.) orgánico de las principales zonas cafetaleras del Perú. Scientia Agropecuaria, 9(2), 177-187.

doi: http://dx.doi.org/10.17268/sci.agropecu.2018.02.02

Figueroa-Benavente, R., Felix-Quillama, R. E., \& Figueroa-Serrano, R. F. (2018). Cambio Climatico y Producción del Cafe en el Valle de la Convencion, Cusco: 2000-2016. Obtenido de: http://13.90.99.62/handle/uglobal/21

Galindo, L. M., Alatorre-Bremont, J. E., \& Reyes-Martínez, O. (2015). Adaptación al cambio climático a través de la elección de cultivos en Perú. El trimestre económico, 82(327), 489519. Obtenido de: http://www.scielo.org.mx/scielo.php?pid=S2448$\underline{718 X 2015000300489 \& \text { script=sci_abstract\&tlng=en }}$

Gómez, G., Fernando, L., \& Zúñiga Escobar, O. (2015). Methodology for assessing vulnerability to climate change of rural househods of Cali hillside districts using fuzzy logic. Revista de Ciencias, 19(1), 89-105. Obtenido de: http://www.scielo.org.co/scielo.php?script=sci_arttext\&pid=S0121-19352015000100007

Granados-Montero, M., Avelino, J., Arauz-Cavallini, F., Castro-Tanzi, S., \& Ureña, N. (2020). Leaf litter and Mycena citricolor inoculum on the American leaf spot epidemic. Agronomía Mesoamericana, 31(1), 77-94. doi: http://dx.doi.org/10.15517/am.v31i1.36614

Gutiérrez Cedillo, J. G., Aguilera Gómez, L. I., \& González Esquivel, C. E. (2008). Agroecología y sustentabilidad. Convergencia, 15(46), 51-87. Obtenido de:

http://www.scielo.org.mx/scielo.php?script=sci_arttext\&pid=S1405-14352008000100004

INEI. (2012). IV Censo Nacional Agropecuario 2012. Obtenido de: http://siea.minagri.gob.pe/siea/?q=iv-censo-nacional-agropecuario-2012

Jha, S., C.M. Bacon, S.M. Philpott, R.A. Rice, V.E. Méndez, \& Läderach, P. (2011). A review of ecosystem services, farmer livelihoods, and value chains in shade coffee agroecosystems. In: W.B. Campbell, and S. López-Ortíz, editors, Agroecology: Integrating agriculture, conservation and ecotourism: examples from the field-present status and future prospectus. 
Springer, Dordrecht, HOL. p. 141-208. doi: https://doi.org/10.1007/978-94-007-1309-3_4.

Jarvis, A., Lane, A., Hijmans, R. (2008). The effect of climate change on crop wild relatives. Agr., Ecosyst. Environm 126(1):13-23. doi:https://doi.org/10.1016/j.agee.2008.01.013

Jezeer, R. E., Verweij, P. A., Boot, R. G., Junginger, M., \& Santos, M. J. (2019). Influence of livelihood assets, experienced shocks and perceived risks on smallholder coffee farming practices in Peru. Journal of environmental management, 242, 496-506. Doi: https://doi.org/10.1016/j.jenvman.2019.04.101

Locatelli, B., V. Evans, D.A. Wardell, A. Andrade, and R. Vignola (2011). Forests and climate change in Latin America: linking adaptation and mitigation. Forests 2:431-450. doi:10.3390/f2010431

Lechthaler, F., \& Vinogradova, A. (2017). The climate challenge for agriculture and the value of climate services: Application to coffee-farming in Peru. European Economic Review, 99, 530. doi:https://doi.org/10.1016/j.euroecorev.2017.06.006

Libert-Amico, A., \& Paz-Pellat, F. (2018). Del papel a la acción en la mitigación y adaptación al cambio climático: la roya del cafeto en Chiapas. Madera y bosques, 24(5). doi: http://dx. doi. org/10.21829/myb. 2018.2401914.

MINAM. (2015). Datos de bosque y pérdida de bosque 2001-2015. Recuperado de: http://geobosques.minam. gob.pe/geobosque/view/perdida.php

MINAM. (2016). La conservación de bosques en el Perú (2011-2016). Conservando los bosques en un contexto de cambio climático como aporte al crecimiento verde. Lima: Minam.

Obtenido de: http://www.minam.gob.pe/informessectoriales/wpontent/uploads/sites/112/2016/02/11-La-conservaci\%C3\%B3n-de-bosques-en-elPer\%C3\%BA.pdf

Mora, J. E. G. (2019). Indicadores bióticos del cambio climático: casos granadilla y café. YachayRevista Científico Cultural, 8(1), 522-529. doi: https://doi.org/10.36881/yachay.v8i1.130

Montiel-González, I., Martínez-Santiago, S., Santos, A. L., \& Herrera, G. G. (2017). Impacto del cambio climático en la agricultura de secano de Aguascalientes, México para un futuro cercano (2015-2039). Revista Chapingo Serie Zonas Áridas, 16(1), 1-13. doi: 10.5154/r.rchsza.2017.01.001.

Ovalle-Rivero, O., Laderach, P., Bunn, C., Obersteiner, M., Schroth, G. (2015). Projected shifts in Coffea arabica suitability among major global producing regions due to climate change, Plos 
One 10(4): 1-13. https://doi.org/10.1371/journal.pone.0124155

Pérez-Pérez, E. F., \& Villafuerte-Solís, D. (2018). Efectos del mercado desregulado sobre los campesinos productores de café de Los Altos de Chiapas: el caso de UCIPA. LiminaR, 16(1), 134-149. doi: http://dx. doi.org/10.29043/liminar. v16i1. 569.

Quipuscoa-Silvestre, V., Dillon, M. O., Treviño-Zevallos, Í., Balvin-Aguilar, M., Mejía-Rios, A., Ramos-Aranibar, D., \& Montesinos-Tubée, D. (2019). Impacto de los cambios climáticos y uso de suelo, en la distribución de las especies de géneros endémicos de Asteraceae de Arequipa. Arnaldoa, 26(1), 71-96. doi: http://dx.doi.org/10.18781/r.mex.fit.1612-7.

Quispe-Apaza, C. S., Mansilla-Samaniego, R. C., López-Bonilla, C. F., Espejo-Joya, R., Villanueva-Caceda, J., \& Monzón, C. (2017). Diversidad genética de Hemileia vastatrix de dos zonas productoras de café en el Perú. Revista mexicana de fitopatología, 35(3), 418-436. doi: http://dx.doi.org/10.18781/r.mex.fit.1612-7

Ramírez, C., Daza, J., \& Peña, A. J. (2015). Tendencia anual de los grados día cafeto y los grados día broca en la región andina ecuatorial de Colombia. Ciencia y Tecnología Agropecuaria, 16(1), 51-63. doi: https://doi.org/10.21930/rcta.vol16_num1_art:379.

Rebolledo, W., Luis, M. L., Peña, A. S. \& Rodríguez, I. (2016). Regulación biológica de Hypothenemus hampei (Ferrari) en café (Coffea arabica L.) con el uso de estrados vegetales en el municipio Junín, Táchira. Universidad \& Ciencia 5(2). Obtenido de: http://revistas.unica.cu/index.php/uciencia/article/view/244

Robiglio, V., Baca, M. G., Donovan, J., Bunn, C., Reyes, M., Gonzáles, D., \& Sánchez, C. (2017). Impacto del cambio climático sobre la cadena de valor del café en el Perú. https://scholar.google.es/scholar?hl=es\&as_sdt=0\%2C5\&q=Impacto+del+cambio+clim\%C3 \%A1tico+sobre+la+cadena+de+valor+del+caf\%C3\%A9+en+el+Per\%C3\%BA.\&btnG=

Schroth, G., Laderach, P., Dempewolf, J., Philpott, S., Haggar, J., Eakin, H., Castillejos, T., Garcia, M.J., Pinto, L.S., Hernandez, R., Eitzinger, A., \& Ramirez-Villegas, A. (2009). Towards a climate change adaptation strategy for coffee communities and ecosystems in the Sierra Madre de Chiapas, Mexico. Mitigation and Adaptation Strategies for Global Change 14(7): 605-625. doi: https://doi.org/10.1007/s11027-009-9186-5.

Sánchez-Castillo, V., Avendaño-Pizo, Y., Gaviria-Astudillo, A., \& Gómez, C. (2018). Cambio climático y café (Coffea arábica) en Acevedo, Huila: una lectura desde sus cultivadores. $I+D$ Revista de investigaciones, 12(2), 59-69. Obtenido de: 
http://udi.edu.co/revistainvestigaciones/index.php/ID/article/view/187

Tobar, D. E., \& Ibrahim, M. (2010). ¿ Las cercas vivas ayudan a la conservación de la diversidad de mariposas en paisajes agropecuarios?. Revista de Biología Tropical, 58(1), 447-463. https://www.scielo.sa.cr/scielo.php?script=sci_arttext\&pid=S0034-77442010000100032

Vignola, R., C.A Harvey, P. Bautista-Solis, J. Avelino, B. Rapidel, C. Donatti, and R. Martinez. (2015). Ecosystem-based adaptation for smallholder farmers: Definitions, opportunities and constraints. Agric. Ecosyst. Environ. 211:126.132. doi:10.1016/j.agee.2015.05.013.

Vallejo, C., Chacón, M., \& Cifuentes, M. (2016). Sinergias entre adaptación y mitigación del cambio climático (SAM) en los sectores agrícola y forestal. Concepto y propuesta de acción. Turrialba, Costa Rica: CATIE-USAID. Obtenido de: http://201.207.189.89/bitstream/handle/11554/8249/Sinergias_entre_adaptacion_y_mitigaci on.pdf? sequence $=7 \&$ is Allowed $=\mathrm{y}$

Villers, L., Arizpe, N., Orellana, R., Conde, C., \& Hernández, J. (2009). Impactos del cambio climático en la floración y desarrollo del fruto del café en Veracruz, México. Interciencia, 34(5), 322-329.obtenido de: https://www.redalyc.org/pdf/339/33911403004.pdf

Velásquez, O., Yesenia, N., \& Pombo, O. A. (2019). La evolución tecnológica y la percepción de la calidad ambiental de los caficultores de El Águila, Valle del Cauca, Colombia. Frontera norte, 31. Doi: http://dx.doi.org/10.33679/rfn.v1i1.1988.

Wayne, H. (2002). Implications of atmospheric and climatic change for crop yield and water use efficiency. Crop Science, 42(1):131- 140. doi: https://doi.org/10.2135/cropsci2002.1310

Wintgens, J. N. (2004). Coffee: Growing, Processing, Sustainable Production. A Guidebook for Growers, Processors, Traders, and Researchers. Weinheim, Alemania: Wiley-VCH Verlag. https://www.cabdirect.org/cabdirect/abstract/20053043070.

Zárate-Malpica, A. H., \& Miranda-Zambrano, G. A. (2016). Impacto del cambio climático en la seguridad alimentaria en zonas campesinas vulnerables de los Andes del Perú. Revista mexicana de ciencias agrícolas, 7(1), 71-82. obtenido de: http://www.scielo.org.mx/scielo.php?script=sci_arttext\&pid=S2007-09342016000100071. 